Pacific Journal of Mathematics

THE POLYNOMIAL OF A NON-REGULAR DIGRAPH 


\title{
THE POLYNOMIAL OF A NON-REGULAR DIGRAPH
}

\author{
W. G. BRIDGES
}

This paper studies the matrix equation $f(Z)=D+\lambda J$ where $f$ is a polynomial, $Z$ a square $(0,1)$-matrix, $D$ is diagonal, $\lambda \neq 0$ and $J$ is the matrix of ones. If $Z$ is thought of as the incidence matrix of a digraph $G$, the equation implies various path length properties for $G$. It is shown that such a graph is an amalgamation of regular subgraphs with similar path length properties. Necessary and sufficient parameter conditions on the matrix $Z$ are given in order that it satisfy such an equation for a fixed polynomial $f$ and all non-regular digraphs corresponding to quadratic polynomials $f$ are found.

1. Introduction. The concept of the polynomial of a graph was introduced by Hoffman [3] for regular, connected, non-oriented graphs, and discussed by Hoffman and McAndrew [4] for regular directed graphs. If $A$ is the adjacency matrix of such a graph $G$, the polynomial of $G$ is taken to be the polynomial $p(x)$ of least degree with $p(A)=J$, the matrix of ones. In extending this notion to nonregular, directed graphs we are concerned with the matrix equation

$$
f(Z)=D+\lambda J
$$

where $f$ is a polynomial, $Z$ a square $(0,1)$ matrix, $D$ a diagonal matrix and $\lambda \neq 0$. Given (1.1) the conditions: (a) $Z$ has constant row sums; (b) $D$ is a scalar matrix; (c) $Z$ has constant line sums; and (d) The graph of $Z$ is regular; are easily seen to be equivalent. The regular case of (1.1) embraces such studies as the $(v, k, \lambda)$-problem [7], $(n, k, \lambda)$-systems on $k$ and $k+1$ [1], Moore graphs [4], strongly regular graphs $[9,10,11,12]$ and even the algebraic studies of central groupoids and universal algebras ]2], [6].

In [8] Ryser opens the non-regular question by considering (1.1) with $f(x)=x^{2}$, and finding all nonregular solutions.

The case in which $f$ of (1.1) has degree two is particularly inter-

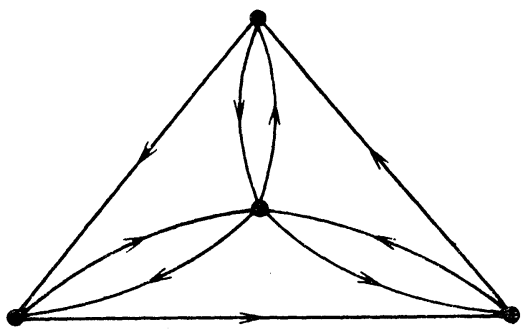

FIG. 1 
esting. Here we are studying directed graphs with the feature that there are constants $\mu_{0}, \mu_{1}$ so that for distinct points $p_{i}, p_{j}$ the number of directed paths from $p_{i}$ to $p_{j}$ of length two is $\mu_{1}$ or $\mu_{0}$ depending on whether or not there is a directed edge from $p_{i}$ to $p_{j}$. For example the digraph $G$ of figure one has this property with $\mu_{1}=$ $1, \mu_{0}=2$. Its adjacency matrix is given by

$$
Z=\begin{array}{llll}
0 & 1 & 1 & 1 \\
1 & 0 & 1 & 0 \\
1 & 0 & 0 & 1 \\
1 & 1 & 0 & 0
\end{array}
$$

satisfying

$$
Z^{2}+Z=\operatorname{diag}(1,-1,-1,-1)+2 J
$$

It is easy to see how various graph theoretic properties expressible in terms of path lengths can be reflected in (1.1) by suitably choosing the polynomial $f$.

In the next section we give a general structure result for matrices satisfying (1.1) showing them to be an "amalgamation" of regular solutions to similar equations. We refine this structure result by considering certain special polynomials $f(x)$. Finally we determine all non-regular quadratic graphs, i.e., those whose adjacency matrix satisfies (1.1) with $f$ a quadratic polynomial.

Throughout $J$ will denote a matrix of ones, $I$ an identity matrix and subscripts on these symbols will denote their orders when necessary.

2. The structure of $f$-graphs. Let $G$ be a directed graph (loops allowed) on $n$ vertices $\{1, \cdots, n\}$ with adjacency matrix $Z=\left(z_{i j}\right)\left(z_{i j}=\right.$ 1 if there is an edge from $i$ to $j$ and $z_{i j}=0$ otherwise). Further let $f$ be a monic polynomial with $f(0)=0$. We say that $G$ is an $f$-graph or that $Z$ carries an $f$-graph if there is a diagonal matrix $D$ and a number $\lambda \neq 0$ so that

$$
f(Z)=D+\lambda J
$$

We shall sometimes say, for $Z$ with constant line sums, that $Z$ carries a degenerate $f$-graph if $f(Z)$ is a scalar matrix.

Let $Z_{i}$ be a square $(0,1)$-matrix of order $n_{i}$ for $i=1,2, \cdots, t$ and $n=\sum_{i=1}^{t} n_{i}$. We define the complementary direct sum (c.d.s) of the matrices $Z_{i}$ by

$$
\text { c.d.s. }\left(Z_{i} \mid i=1, \cdots, t\right) \equiv J_{n}-\sum_{i=1}^{t} \oplus\left(J_{n_{i}}-Z_{i}\right)
$$


where $\sum \oplus$ denotes the usual direct sum.

Finally since evidently for $P$ a permutation matrix with transpose $P^{t}$ the matrix $P Z P^{t}$ will satisfy (2.1) if $Z$ does we define $A$ and $B$ to be equivalent, $A \cong B$, if there is a permutation matrix $P$ so that $A=P B P^{t}$. The relevance of these definitions will be clear from the following theorem.

THEOREM 2.1. Let $Z$ be a $(0,1)$-matrix of order $n$. Suppose $Z$ carries an f-graph. Then we have

$$
Z \cong \text { c.d.s. }\left(Z_{i} \mid i=1, \cdots, t\right)
$$

where the matrices $Z_{i}$ of order $n_{i}$ have constant line sums $r_{i}$ with $r_{i}-n_{i} \neq r_{j}-n_{j}$ for $i \neq j$ and each $Z_{i}$ carries an f-graph (possibly degenerate).

We delay the proof of this elementary observation as we can say considerably more. We only state Theorem 2.1 in order to put the next theorem in proper perspective.

THEOREM 2.2. Let $Z_{i}$ be a $(0,1)$-matrix of order $n_{i}$ with constant line sums $r_{i}$ for $i=1, \cdots, t$. For $i \neq j$ suppose $r_{i}-n_{i} \neq r_{j}-n_{j}$. Put

$$
\begin{gathered}
Z=\text { c.d.s. }\left(Z_{i} \mid i=1,2, \cdots, t\right) \\
r_{i j}=\left(r_{i}-n_{i}\right) \delta_{i j}+n_{j}(i, j=1, \cdots, t)
\end{gathered}
$$

$\left(\delta_{i j}\right.$ denoting Kronecker's delta) and

$$
R=\left(r_{i j}\right) \text {. }
$$

Finally left $f$ be a monic polynomial of degree at least two with $f(0)=0$.

Then $Z$ carries an $f$-graph if and only if there exist constants $b$ and $\lambda$ with $\lambda \neq 0$, and numbers $d_{i}, \lambda_{i}(i=1, \cdots, t)$ so that

$$
\begin{aligned}
& d_{i}=\lambda\left(r_{i}-n_{i}\right)+b(i=1, \cdots, t) \\
& f\left(Z_{i}\right)=d_{i} I+\lambda_{i} J_{n_{i}}(i=1, \cdots, t)
\end{aligned}
$$

and

$$
f(R)=\lambda R+b I \text {. }
$$

We proceed to prove both theorems. It follows from (2.1) that $Z$ commutes with $D+\lambda J$ if $Z$ carries an $f$-graph. Denoting $D=$ $\operatorname{diag}\left(d_{1}, \cdots, d_{n}\right)$, the row sums of $Z$ by $\rho_{i}(i=1, \cdots, n)$ and the column sums by $\sigma_{j}(j=1, \cdots, n)$ this fact may be expressed as 


$$
z_{i j}\left(d_{i}-d_{j}\right)=\lambda\left(\rho_{i}-\sigma_{j}\right) .
$$

We thus have $\rho_{i}=\sigma_{i}$ and if we permute the rows of $Z$ so that rows with equal sum occur together and then perform the corresponding column permutations we obtain $P Z P^{t}$ with say rows $1,2, \cdots, n_{1}$ of equal sum and then rows $n_{1}+1, n_{1}+2, \cdots, n_{1}+n_{2}$ of equal sum etc. Let $Z_{i}$ denote the principal submatrix of $P Z P^{t}$ on the lines $n_{i-1}+1, \cdots, n_{i-1}+n_{i}\left(n_{0} \equiv 0\right)$. We assert that no entry of $Z$ outside one of these $Z_{i}$ can be a zero for then from (2.10) we have $\rho_{i}=\sigma_{j}=$ $\rho_{j}$ contrary to our grouping of the rows. Thus we have established (2.3) where $t$ is the number of distinct row sums of $Z$. It is immediate that $Z_{i}$ of order $n_{i}$ has constant line sums say $r_{i}(i=1, \cdots, t)$ and that $r_{i}-n_{i} \neq r_{j}-n_{j}$ for $i \neq j$ since $\rho_{j}=r_{i}+n-n_{i}$.

We now deal with $Z$ in the equivalent c.d.s. form and take $t>1$ lest $Z$ be regular. Note from (2.10) that the diagonal matrix $D$ has different entries in positions corresponding to different blocks $Z_{i}$. It is further clear from (2.10) that the entries in $D$ in positions corresponding to the same block $Z_{i}$ are the same. We therefore revise our notation so that $d_{1}, d_{2}, \cdots, d_{t}$ denote the distinct diagonal entries of $D, d_{i}$ occurring $n_{i}$ times. Then (2.10) says that the points $\left(d_{i}, r_{i}-\right.$ $n_{i}$ ) for $i=1,2, \cdots, t$ lie on the line $y=\lambda x+b$ for some constant $b$. [Note it is the choice of normalization $(f(0)=0)$ which brings the constant $b$ into play. We could, of course, force $b=0$ by altering the constant term of $f(x)$.]

We now investigate the powers of the matrix $Z$ in c.d.s. form and assert that in block form, the $(i, j)^{\text {th }}$ block of size $n_{i} \times n_{j}$, we have

$$
Z^{k}=\left[B_{i j}\right](i, j=1, \cdots, t)
$$

where

$$
B_{i j}=\delta_{i j} Z_{i}^{k}+g_{i j}^{(k)} J_{n_{i} \times n_{j}} \cdot
$$

The numbers $g_{i j}^{(k)}$ are given by

$$
g_{i i}^{(1)}=0, g_{i j}^{(1)}=1 \text { for } i \neq j
$$

and for $k>1$

$$
g_{i j}^{(k)}=\sum_{e=1}^{t} n_{e} g_{e j}^{(k-1)}+\left(r_{i}-n_{i}\right) g_{i j}^{(k-1)}+r_{j}^{k-1}\left(1-\delta_{i j}\right) .
$$

This claim is easily verified inductively.

We introduce the following notational convention: If $p(x)=$ $\sum_{i=1}^{m} b_{i} x^{i}$ is a polynomial in $x$ and $\alpha^{(k)}$ is a symbol in use with superscripts then by $\hat{p}(\alpha)$ we will mean the expression $\hat{p}(\alpha) \equiv \sum_{i=1}^{m} b_{i} \alpha^{(i)}$, 
using the iterates of $\alpha$ in place of the powers of $x$. Then from (2.12) - (2.13) we have

$$
f(Z)=\left[C_{i j}\right](i, j=1, \cdots, t)
$$

$C_{i j}$ being an $n_{i} \times n_{j}$ block given by

$$
\begin{gathered}
C_{i i}=f\left(Z_{i}\right)+\hat{f}\left(g_{i i}\right) J_{n_{i}}(i=1, \cdots, t) \\
C_{i j}=\hat{f}\left(g_{i j}\right) J_{n_{i} \times n_{j}} i \neq j(i, j=1, \cdots, t) .
\end{gathered}
$$

with

$$
G^{(k)}=\left(g_{i j}^{(k)}\right)(i, j=1, \cdots, t)
$$

we see that the structure of $f(Z)$ depends on the matrix $\hat{f}(G)$. We note, however, from (2.16), (21.7) that if $Z$ carries an $f$-graph

$$
\begin{aligned}
& f\left(Z_{i}\right)+\hat{f}\left(g_{i i}\right) J_{n_{i}}=d_{i} I+\lambda J \text { so that } \\
& f\left(Z_{i}\right)=d_{i} I+\left(\lambda-\hat{f}\left(g_{i i}\right)\right) J_{n_{i}}
\end{aligned}
$$

and the $Z_{i}$ carry $f$-graphs, degenerate should $\lambda=\hat{f}\left(g_{i i}\right)$. We have thus completely proven Theorem 2.1 and continue with the necessity in Theorem 2.2 where we have already established (2.7) and (2.8). To obtain (2.9) we proceed to observe that the recursion (2.24), (2.15) can be written: $G^{(1)}=J-I$ and for $k>1$ :

$$
G^{(k)}=R G^{(k-1)}+(J-I) F^{k-1}
$$

where $F=\operatorname{diag}\left(r_{1}, \cdots, r_{t}\right)$. We obtain an explicit formula for $G^{(k)}$ as follows. Let $E=\operatorname{diag}\left(1 / n_{1}, \cdots, 1 / n_{t}\right)$ and consider

$$
H^{(k)} \equiv\left[R^{k}-F^{k}\right] E, k \geqq 1 \text {. }
$$

We claim that $H^{(k)}$ satisfies the recursion (2.21). For $k=1, H^{(1)}=$ $G^{(1)}=J-I$. Now for $k>1$ we have:

$$
R H^{(k-1)}+(J-I) F^{k-1}=\left[R^{k}-R F^{k-1}\right] E+(J-I) F^{k-1}
$$

so

$$
R H^{(k-1)}+(J-I) F^{k-1}=R^{k} E-\left(R-(J-I) E^{-1}\right) F^{k-1} E .
$$

But $R-(J-I) E^{-1}=F$ so we have $R H^{(k-1)}+(J-I) F^{k-1}=\left[R^{k}-\right.$ $\left.F^{k}\right] E=H^{(k)}$. Thus $H^{(k)}=G^{(k)}$ and

$$
\hat{f}(G)=[f(R)-f(F)] E .
$$

Now if $Z$ is carrying an $f$-graph the off-diagonal entries of $\hat{f}(G)$ are all $\lambda$. Thus the off-diagonal entries of $f(R)$ are given by

$$
f(R)_{i, j}=\lambda n_{j}(i \neq j ; i, j=1, \cdots, t) .
$$


From (2.20) we see that

$$
f\left(r_{i}\right)=d_{i}+\left(\lambda-\hat{f}\left(g_{i i}\right)\right) n_{i}
$$

so that

$$
\hat{f}\left(g_{i i}\right)=\frac{f(R)_{i i}-f\left(r_{i}\right)}{n_{i}}=\frac{d_{i}+\lambda n_{i}-f\left(r_{i}\right)}{n_{i}} .
$$

Since $d_{i}=\lambda\left(r_{i}-n_{i}\right)+b$ we have

$$
f(R)_{i i}=d_{i}+\lambda n_{i}=\lambda r_{i}+b,
$$

and (2.24) and (2.26) establish (2.9).

As to the sufficiency of $(2.7)-(2.9)$ we need only note that (2.23) is a valid expression for any polynomial $f$ and that using (2.7), (2.8) with

$$
\hat{f}(G)=[\lambda R+b I-f(F)] E
$$

we see $\hat{f}(G)_{i j}=\hat{f}\left(g_{i j}\right)=\lambda(i \neq j ; i, j=1, \cdots, t)$ and

$$
\hat{f}\left(g_{i i}\right)=\frac{\lambda r_{i}+b-f\left(r_{i}\right)}{n_{i}}=\frac{d_{i}+\lambda n_{i}-f\left(r_{i}\right)}{n_{i}}
$$

so that in view of $f\left(r_{i}\right)=d_{i}+n_{i} \lambda_{i}$

$$
f\left(Z_{i}\right)+\hat{f}\left(g_{i i}\right) J=d_{i} I+\lambda_{J} .
$$

This completes the proof of Theorems 2.1 and 2.2. An immediate corollary of these results is that we may define the notion of the polynomial of a directed graph if the graph is an $f$-graph for some $f$.

COROLlary 2.3. Let $Z$ carry an f-graph for some $f$. Then there exists a unique monic polynomial $P_{z}(x)$ with $P_{z}(0)=0$ of least degree so that $Z$ carries a $P_{z}$-graph.

Proof. Let $g(x)$ and $h(x)$ be two such polynomials. Suppose

$$
g(Z)=D+\lambda J, h(Z)=H+\mu J
$$

$D, H$ diagonal $\lambda \neq 0, \mu \neq 0$. Then since

$$
(g-h) Z=(D-H)+(\lambda-\mu) J
$$

evidently $Z$ carries a degenerate $(g-h)$-graph, i.e., $\lambda=\mu$. But for suitable constants $b, c$ we have $d_{i}=\lambda\left(r_{i}-n_{i}\right)+b$ and $h_{i}=\mu\left(r_{i}-n_{i}\right)+c$ so that $D-H$ is a scalar matrix and since surely $g$ and $h$ have degree less than that of $Z$ 's minimal polynomial and $g(0)=h(0)=0$ 
we have $g(x) \equiv h(x)$.

Professor Hoffman has observed that the condition that $Z$ carry an $f$-graph for some $f$ can be expressed in terms of the spectra of the matrices $Z_{i}$ of (2.4) and that of the parameter matrix $R$ of (2.6) as follows. For $n_{i}>1$ delete from the eigenvalue set of $Z_{i}$ the line sum $r_{i}$ if $Z_{i}$ is irreducible and call the resultant set $\Lambda_{i}$. Let $\Lambda_{0}$ be the set of eigenvalues of the matrix $R$. Then $Z$ carries an $f$-graph for some $f$ if and only if

$$
\Lambda_{i} \cap \Lambda_{j}=\phi \text { for all } n_{i}, n_{j}>1, i \neq j, i, j=0,1 \cdots, t .
$$

To see this one need only observe that the congruences

$$
\begin{aligned}
& f(x) \equiv d_{i} \bmod \dot{m}_{i}(x) \\
& f(x) \equiv \lambda x+b \bmod q(x)
\end{aligned}
$$

(where $\dot{m}_{i}(x)$ is the minimal polynomial of $Z_{i}$ with $\left(x-r_{i}\right)$ divided out in case $Z_{i}$ is irreducible and $q(x)$ is the minimal polynomial of $R$ ) are satisfied by $f$ so that the $\dot{m}_{i}(x)$ and $q(x)$ cannot share roots. Note that for $\dot{m}_{i}(x)$ and $q(x)$ one needs the fact that $r_{i}-n_{i}=\left(d_{i}-b\right) / \lambda$ is not a root of $q(x)$. The converse statement is also quite immediate in view of Theorem 2.2.

We shall call the matrices $Z_{i}$ (or the obviously associated subgraphs $G_{i}$ ) in the c.d.s. form of $Z$ the regular constituents of $Z$. So $Z$ is regular if it has one constituent and we will call $Z$ near-regular if it has precisely two regular-constituents.

Now the parameter matrix $R(2.6)$ is readily seen to be similar to the symmetric matrix

$$
S=\operatorname{diag}\left(r_{1}-n_{i}, \cdots, r_{t}-n_{t}\right)+\left(\sqrt{n_{i} n_{j}}\right) .
$$

Indeed

$$
R=E^{1 / 2} S E^{-1 / 2}
$$

where $E=\operatorname{diag}\left(1 / n_{1}, \cdots, 1 / n_{t}\right)$. Easily for the $\left(r_{i}-n_{i}\right)$ distinct the matrix $R$ has $t$ distinct real characteristic roots, and that precisely one of these roots is positive. Now if $Z$ carries an $f$-graph we have $f(R)=\lambda R+b I$ so that the minimal polynomial (= characteristic polynomial) of $R$ divides $f(x)-\lambda x-b$. We thus establish

CoROLLARY 2.4. Let $Z$ carry an f-graph with $t$ regular constituents. Then $t \leqq$ degree $f$, and, if equality holds, $f(x)-\lambda x-b$ is the characteristic polynomial of $R$.

The case of equality may occur. For example the matrix 


$$
Z=\begin{array}{llll}
1 & 1 & 1 & 1 \\
1 & 0 & 1 & 1 \\
1 & 1 & 0 & 0 \\
1 & 1 & 0 & 0
\end{array}
$$

has 3 regular constituents and its polynomial is $x^{3}-x^{2}-4 x$. However with various restrictions on $f(x)$ we can make stronger statements about the number of constituents of an $f$-graph.

COROLlaRY 2.5. Suppose $f(x)$ is an even polynomial with nonnegative coefficients. Then a non-regular f-graph is near-regular.

Proof. For suitable $\lambda>0$ and $b$ we have that $q(x)$, the characteristic polynomial of the parameter matrix $R$, devides $g(x)=f(x)-$ $\lambda x-b$. But $g(-x)=f(x)+\lambda x-b$ so that $g$ has at most one negative root. However $q(x)$ has $t-1$ negative roots. Thus $t \leqq 2$.

An interesting class of polynomial graphs are the $x^{r}$-graphs investigated by Ryser [7] for $r=2$. These graphs have the feature that the number of paths of length $r$ joining any two distinct points is constant. The preceding corollary shows that for $r$ even such graphs, if not regular, are near regular. This property extends to all $x^{r}$-graphs.

CoRollary 2.6. A non-regular $x^{r}$-graph $(r \geqq 2)$ is near regular.

Proof. Again $g(x)=f(x)-\lambda x-b=x^{r}-\lambda x-b$ must have at least $t$ distinct real roots, where $t$ is the number of regular constituents of the graph. But $g^{\prime}(x)$ has at most two real roots so that $t \leqq 3$. The following argument suggested by Professor Hoffman shows that $t \neq 3$. Suppose $t=3$. Then $q(x)=x^{3}-a x^{2}+h x-\Delta$ where $a=r_{1}+r_{2}+r_{3}, h=\sum_{i<j}\left(r_{i} r_{j}-n_{i} n_{j}\right)$ and $\Delta=\operatorname{det} R$, is the characteristic polynomial of $R$. Easily $a>0, h<0, \Delta>0$. Since $q(x)$ divides $f(x)-\lambda x-b$ we may write

$$
\left(x^{r}-\lambda x-b\right)=\left(x^{3}-a x^{2}+h x-\Delta\right)\left(x^{r-3}+a_{4} x^{r-4}+\cdots+a_{r-1} x+a_{r}\right) .
$$

Equating coefficients in this identity we obtain

$$
\begin{aligned}
& a_{4}-a=0 \\
& a_{5}-a a_{4}+h=0 \\
& a_{6}-a a_{5}+h a_{4}-\Delta=0 \\
& a_{j+3}-a a_{j+2}+h a_{j+1}-\Delta a_{j}=0(4 \leqq j \leqq r-3) .
\end{aligned}
$$

The relations (2.29) imply for $i=4, \cdots, r$ that $a_{i}>0$. Now equating 
the coefficients of $x^{2}$ we obtain the contradiction

$$
-a a_{r}+h a_{r-1}-\Delta a_{r-2}=0 \text {. }
$$

Of course $r=3$ implies $a=0$.

We remark that the existence of a non-regular $x^{r}$-graph on more than two points $r>2$ remains in question.

Suppose one constituent of an $f$-graph is a (looped or unlooped) point. Then we shall refer to the graph as a (looped or unlooped) cone. For example let $K_{a}$ denote the full (looped) directed clique on a vertices. Take some number say $m>1$ of copies of $K_{a}$ and form the looped cone over this graph with respect to an additional point. The resulting graph is a non-regular $\left(x^{2}-a x\right)$-graph. We shall in fact see in the next section that with one exception all non-regular quadratic graphs are cones.

3. Non-regular quadratic graphs. In this section we determine all non-regular $f$-graphs for $f(x)=x^{2}-a x$. We characterized these graphs in the introduction in terms of path lengths. From Corollary 2.4 we know such graphs are near regular. Our first observation is that there is precisely one such graph which is not a cone.

THEOREM 3.1. Let $Z$ carry a non-regular quadratic graph which is not a cone. Then

10111

01111

$$
Z \cong \begin{array}{lllll}
1 & 1 & 0 & 0 & 1 \\
1 & 1 & 1 & 0 & 0 \\
1 & 1 & 0 & 1 & 0
\end{array}
$$

and carries an $\left(x^{2}+x\right)$-graph.

Proof. Let $Z_{1}, Z_{2}$ of orders $n_{1}, n_{2}$ and line sums $r_{1}$ and $r_{2}$ be the regular constituents of $Z$ and suppose $n_{i}>1 \quad(i=1,2)$. We note from Corollary 2.4 that

$$
r_{1}+r_{2}=\lambda+a .
$$

We first remark that neither constituent can be $J-I$. For suppose $r_{1}=n_{1}-1$. Then from (3.2) $r_{2}=\lambda+a-n_{1}+1$ and we have

$$
f(Z)=\frac{f\left(Z_{1}\right)+n_{2} J}{\lambda_{3} J} \mid \frac{\lambda_{1} J}{f\left(Z_{2}\right)+n_{1} J} .
$$

So with $Z_{1}=J-I$ we have 


$$
n_{1}+n_{2}-a-2=\lambda=r_{2}-a+n_{1}-1
$$

which forces $n_{2}-r_{2}=1=n_{1}-r_{1}$ implying $Z$ is regular. One can similarly eliminate $Z_{i}=J$ for $n_{i}>1$. Consider next the possibility $Z_{1}=I$. From (3.2) and (3.3) we have

$$
\lambda=n_{2}=1+r_{2}-a
$$

so the constituent $Z_{2}$ satisfies

$$
Z_{2}^{2}+\left(n_{2}-r_{2}-1\right) Z_{2}=\left[\left(n_{2}-1\right)\left(r_{2}-\lambda_{2}\right)-\lambda_{2}\right] I+\lambda_{2} J
$$

where $\lambda_{2}=n_{2}-n_{1}$ and the coefficient of $I$ is determined by equating line sums. Since the elements of $Z_{2}^{2}$ cannot exceed $r_{2}$ we have

$$
\left(n_{2}-1\right)\left(r_{2}-\lambda_{2}\right) \leqq\left(n_{2}-1\right) \text { with } r_{2} \geqq \lambda_{2} \text {. }
$$

If $r_{2}=\lambda_{2}+1$ we have $r_{2}=n_{2}-n_{1}+1$ whence $r_{2}-n_{2}=1-n_{1}=$ $r_{1}-n_{1}$ and $Z$ is regular. Thus $r_{2}=\lambda_{2}$ and trace $Z_{2}=0$ with

$$
Z_{2}^{2}+\left(n_{2}-r_{2}-1\right) Z_{2}=r_{2}(J-I) .
$$

Now row $i$ and column $i$ of $Z_{2}$ are different while $z_{i j}=0$ forces row $i$ equal column $j$. Hence there is at most one off diagonal zero in any row of $Z_{2}$ and $r_{2}=n_{2}-2, a=-1$. It is then almost immediate from (3.4) that $r_{2}=1, n_{2}=3$ and

$$
Z_{2} \cong \begin{array}{lll}
0 & 0 & 1 \\
1 & 0 & 0 \\
0 & 1 & 0
\end{array} .
$$

Then $\lambda_{2}=n_{2}-n_{1}=1$ so $n_{1}=2$ and we obtain (3.1). We. now suppose neither constituent is a point, $J-I$ or $I$ and assert:

$$
\begin{aligned}
& \lambda+n+a-2\left(r_{1}+n_{2}\right) \geqq 0 \\
& \lambda+n+a-2\left(r_{2}+n_{1}\right) \geqq 0
\end{aligned} .
$$

This can be seen by considering $(0,-1)$-matrix $Z-J$. The quantities (3.5) are off diagonal entries in $f(Z-J)$ and for $Z_{i} \neq I$ these entries are nonnegative. But in view of (3.2) adding the entries in (3.5) gives zero.

Thus

$$
\begin{aligned}
& r_{1}=\frac{\lambda+n_{1}-n_{2}+a}{2}=\frac{\lambda_{1}+n_{1}+a}{2} \\
& r_{2}=\frac{\lambda+n_{2}-n_{1}+a}{2}=\frac{\lambda_{2}+n_{2}+a}{2}
\end{aligned}
$$

where $\lambda_{i}=\lambda+n_{i}-n, f\left(Z_{i}\right)=d_{i} I+\lambda_{i} J$ (see 3.3). Now $f\left(r_{i}\right)=d_{i}+$ 
$n_{i} \lambda_{i}$ gives $d_{i}=\left(r_{i}-\lambda_{i}\right)\left(n_{i}-r_{i}\right)$ and if $Z_{i}$ has a zero in diagonal position we can assert that $d_{i}+\lambda_{i} \leqq r_{i}$. Whence

$$
\left(r_{i}-\lambda_{i}\right)\left(n_{i}-r_{i}\right) \leqq\left(r_{i}-\lambda_{i}\right),
$$

forcing $r_{i}=\lambda_{i}$ and $a=\lambda_{i}-n_{i}=r_{i}-n_{i}$. Thus $Z$ is regular unless trace $\left(Z_{i}\right)=n_{i}$. But in that event we have $d_{i}+\lambda_{i}+a \leqq r_{i}$ which, viewed with (3.6) implies $d_{i} \leqq n_{i}-r_{i}$ or

$$
\left(r_{i}-\lambda_{i}\right)\left(n_{i}-r_{i}\right) \leqq\left(n_{i}-r_{i}\right) \text {. }
$$

Hence $r_{i}=\lambda_{i}, \lambda_{i}+1$. From (3.6) if we are to avoid $r_{1}-n_{1}=$ $r_{2}-n_{2}$ we conclude $r_{1}=\lambda_{1}, r_{2}=\lambda_{2}+1$ with $a=r_{1}-n_{1}=r_{2}-n_{2}+1$. Now (3.3) and (3.2) imply $\lambda=\lambda_{1}+n_{2}=\lambda_{2}+n_{1}=r_{1}+r_{2}-a$ and hence $a=r_{1}-n_{1}+1=r_{1}-n_{1}$ which contradiction completes the proof.

As all remaining non-regular quadratic graphs are cones they fall naturally into two classes: looped and unlooped. We determine these classes separately in the next two theorems.

THEOREM 3.2. Let $G$ be a non-regular $\left(x^{2}-a x\right)$-graph which is the looped cone over $G_{1}$ carried by the matrix $Z_{1}$. Then one of the following holds:

(i) $a$ is a positive integer and

$$
Z_{1} \cong \sum \oplus J_{a}
$$

(ii) $a=1$ and

$$
Z_{1} \cong \begin{array}{rrr}
1 & 1 & 0 \\
0 & 1 & 1 \\
1 & 0 & 1
\end{array}
$$

(3.10) (iii) $a=0, Z_{1}=0$.

Proof. The matrix $Z_{1}$ with line sums $r_{1}$ must satisfy

$$
Z_{1}^{2}-a Z_{1}=d_{1} I+\left(r_{1}-a\right) J
$$

with $d_{1}=\left(r_{1}-n_{1}\right)\left(r_{1}-a\right)$ [See (3.3)]. Note here that if $r_{1}=n_{1}-1$ then $Z_{1}=J-P$ for $P$ a permutation. Now (3.11) will force $n_{1}=3$ and $P$ carrying either of the cycles (123) or (132) yielding (3.9) of the theorem or $n_{1}=2, a=1$ of case (i) - (3.8). Hence we take $1 \leqq r_{1} \leqq n_{1}-2$. We must have $r_{1} \geqq a$ since $r_{1}<a$ implies $d_{1}+\left(a_{1}-a\right)>0$ and the entries of $Z_{1}^{2}$ do not exceed $r_{1}$. We further assert that $r_{1}=a$ only for the family (i) of the theorem for here $Z_{1}^{2}=r_{1} Z_{1}$.

Quite generally from (3.11) we see that $z_{i j}=1, i \neq j$, implies 
row $i$ equal to column $j$. Further if some $z_{i i}=0$ we have

$$
d_{1}+\left(r_{1}-a\right)=\left(r_{1}-n_{1}+1\right)\left(r_{1}-a\right) \geqq 0,
$$

and since $r_{1} \geqq a$ we must conclude that $r_{1}=a$ and obtain the family (i). The remaining candidates for $Z_{1}$ have trace $n_{1}$. If for some $i$, row $i$ and column $i$ are equal then evidently $d_{1}=0$ and $r_{1}=a$ again. But we still have that row $i$ and column $j$ are indentical if $z_{i j}=1$ for $i \neq j$. To avoid an occurrence of row $i$ equals column $i$ easily $r_{1}=1,2$. In the former instance $Z_{1}=I$ (of family (i)) in the latter since $i \neq j$ and $z_{i j}=0$ forces row $i$ and column $j$ to meet in a 2- $a$ positions we deduce that $a=1, n_{1}=3=r_{1}+1$ and we have case (ii).

Finally we treat the case of a non-looped cone. There are several such graphs as the next theorem shows.

THEOREM 3.3. Let $G$ be a non-regular $\left(x^{2}-a x\right)$-graph which is a non-looped cone over $G_{1}$ carried by $Z_{1}$. Then one of the following holds.

(i) $a=0$ and $Z_{1}$ is a symmetric permutation matrix or $Z_{1}=0$.

010

(ii) $a=-1$ and $Z_{1} \cong 001$

100

(iii) $a=2$ and

$$
Z_{1} \cong\left(\begin{array}{llllllll}
1 & 1 & 1 & 1 & 0 & 0 & 0 & 0 \\
1 & 1 & 0 & 0 & 0 & 0 & 1 & 1 \\
1 & 1 & 1 & 1 & 0 & 0 & 0 & 0 \\
0 & 0 & 1 & 1 & 1 & 1 & 0 & 0 \\
0 & 0 & 0 & 0 & 1 & 1 & 1 & 1 \\
0 & 0 & 1 & 1 & 1 & 1 & 0 & 0 \\
1 & 1 & 0 & 0 & 0 & 0 & 1 & 1 \\
0 & 0 & 0 & 0 & 1 & 1 & 1 & 1
\end{array}\right)
$$

(iv) $a=+1$ and $Z_{1}$ is equivalent to one of the following six matrices:
10110
0111100
$\begin{array}{lllllll}0 & 1 & 1 & 1 & 0 & 0 & 0\end{array}$
$\begin{array}{lllll}0 & 1 & 0 & 1 & 1\end{array}$
$\begin{array}{llllll}1 & 1 & 0 & 0 & 0 & 1\end{array}$
$\begin{array}{lllllll}0 & 1 & 0 & 1 & 1 & 0 & 0\end{array}$

(a) 10101

(b) 01011100

(c) $0 \begin{array}{lllllll}0 & 1 & 0 & 0 & 0 & 1\end{array}$

11010

$\begin{array}{llllll}0 & 0 & 1 & 1 & 1 & 0\end{array}$

$\begin{array}{lllllll}0 & 0 & 1 & 1 & 0 & 1 & 0\end{array}$

011101

$\begin{array}{llllll}1 & 0 & 0 & 0 & 1 & 1\end{array}$

$\begin{array}{lllllll}1 & 0 & 0 & 0 & 1 & 0 & 1\end{array}$

1000011

10000110

10000011 

11110000
11110000
111100
$\begin{array}{llllll}1 & 1 & 0 & 0 & 0 & 1\end{array}$
01110011
$\begin{array}{llllll}1 & 1 & 0 & 0 & 1 & 1\end{array}$
$\begin{array}{lllllllllll}0 & 0 & 1 & 1 & 1 & 0\end{array}$
$\begin{array}{llllll}1 & 0 & 0 & 1 & 0 & 1\end{array}$
(e)
$\begin{array}{llllllllllllll}0 & 0 & 1 & 1 & 1 & 1 & 0\end{array}$
$\begin{array}{llllllllll}0 & 1 & 1 & 0 & 1 & 0\end{array}$
0101011101
1111100
(f)
$\begin{array}{lllllll}0 & 0 & 1 & 1 & 1 & 1\end{array}$
11000110
$\begin{array}{llllll}1 & 1 & 0 & 0 & 1 & 1\end{array}$
$\begin{array}{llllll}0 & 0 & 0 & 1 & 1 & 1\end{array}$
1000101011
0001111
101010101

(d)

(v) $a+2$ is a positive integer and

(a) $Z_{1} \cong \sum \oplus(J-I)_{a+2}$ or

$$
\text { (b) } Z_{1} \cong\left(\begin{array}{c|c|c}
0 & & \\
: & J_{a+2} & 0_{a+2, a+1} \\
\hline 0 & & \\
\hline \dot{1} & & \\
: & 0_{a+2} & J_{a+2, a+1} \\
\dot{1} & &
\end{array}\right)
$$

Proof. We have

$$
Z_{1}^{2}-a Z_{1}=d_{1} I+\left(r_{1}-a-1\right) J
$$

with

$$
d_{1}=\left(r_{1}-a\right)\left(r_{1}-n_{1}\right)+n_{1}=\left(r_{1}-a-1\right)\left(r_{1}-n_{1}\right)+r_{1}
$$

using (3.3) and $f\left(r_{1}\right)=d_{1}+n_{1}\left(r_{1}-a-1\right)$. We note that $r_{1}=1$ easily gives $a=0$ with $Z_{1}$ a symmetric permutation or $a=-1$ with $Z_{1}$ carrying either of the three cycles (123) or (132). We also note that the preceding Theorm 3.2 finds all candidates here with trace zero. For then $W=Z+I$ carries a non-regular $\left(x^{2}-(a+2) x\right)$-graph with a looped point as one constituent. These observations give cases (ii) and $(v a)$ of the present theorem as the only $Z_{1}^{\prime}$ s with trace $Z_{1}=0$. We further remark that $Z_{1} \neq J$ and that the examples $(v a)$ are characterized by $r_{1}=a+1$. For in this case $d_{1}=r_{1}$ and $Z_{1}^{2}-a Z_{1}=$ $(a+1) I$, so that if $Z_{1}$ has a diagonal entry equal to one a cannot be positive since the entries of $Z_{1}^{2}$ do not exceed $a+1$. Thus $a=0, r_{1}=1$ discussed above, or $a=-1, Z_{1}=0$, case $v$ with $a=-1$. The remaining possibilities have trace $Z_{1}=0$ and are also discussed above.

We have to consider then trace $Z_{1}$ positive and easily here:

$$
a+1<r_{1}<n_{1}-1 \text {. }
$$

We first suppose some $Z_{i i}=0$. With $Z_{1}^{2}=\left(Q_{i j}\right)$ we have 


$$
Q_{i i}=\left(r_{1}-a-1\right)\left(r_{1}-n_{1}+1\right)+r_{1} \text {. }
$$

But surely $r_{1}-Q_{i i} \leqq n_{1}-r_{1}-1$ so that (3.15) implies

$$
\left(n_{1}-r_{1}-1\right)\left(r_{1}-a-1\right) \leqq\left(n_{1}-r_{1}-1\right) \text {. }
$$

Thus $r_{1}=a+2$ and we may suppose $a \geqq 0$. Now (3.12) will become

$$
Z_{1}^{2}-a Z_{1}=\left[2(a+2)-n_{1}\right] I+J \text {. }
$$

Take

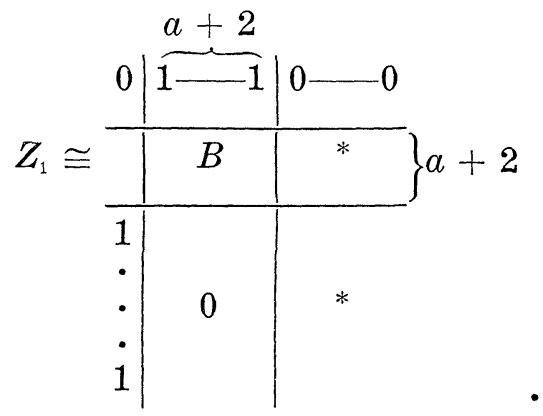

The matrix $B$ of (3.18) has column sums $(a+1)$ and so contains $(a+2)$ zeros. Thus $B$ has a row with at most one zero. This row will meet any column of $B$ in at least $a$ positions. If this row has a zero in an off-diagonal position we have $a=0,1$. If this row has its single zero on the diagonal, say $b_{11}=Z_{22}=0$ then $Z_{21}=1$ so that row 2 of $Z_{1}$ can meet column 3 of $Z_{1}$ in the proper number of positions $\left(z_{23}=1 \Rightarrow Q_{23}=a+1\right)$. But then $Q_{21}=a+1=2 a+4-n_{1}$ so $n_{1}=$ $a+3$ and $Z$ would be regular with $r_{i}-n_{i}=-1$. Thus unless $B$ has a row of all ones $a=0,1$. We consider the case that $B$ indeed has a row of all ones.

Placing this row initially in $B$ and maintaining equivalence a look at row two and column one of $Z_{1}$ gives $n_{1}=2(a+2), Z_{1}^{2}-a Z_{1}=J$. Recall we are avoiding a row in $B$ with precisely one zero, so that $B$ has a row with at least two zeros. This row in $Z_{1}$ has a one in its first position and by checking the row and column through its off-diagonal zero we see this row in $B$ has at most one nonzero entry. This row accounts for at least $a+1$ of the zeros of $B$. Were the remaining zero not in this row we would have a row in $B$ with precisely one zero. Thus $B$ has a zero row and all other rows are full. This gives $Z_{1}$ the form of case $\mathrm{v}$.

We are left with the cases $a=0,1$ and note from (3.17) that $4 \leqq n_{1} \leqq 7$. With $a=0, n_{1}=4,5$ and $n_{1}=5$ gives trace $\left(Z_{1}\right)=0$ while $\mathrm{n}_{1}=4$ gives $(v b)$ with $a=0$. The choice $a=1, n_{1}=5$ is easily eliminated and, for $n_{1}=6$, one obtains $(i v b)$ and $(v b)$. Finally 
with $n_{1}=7$ the matrix (ivc) is obtained after some work.

As this discussion was on the assumption of some $z_{i i}=0$ we are left to investigate those $Z_{1}$ satisfying (3.12), (3.13) and trace $Z_{1}=$ $n_{1}$. To that end we investigate $W=Z_{1}-I$. Evidently

$$
\mathrm{W}^{2}+(2-a) W=\left(d_{1}+a-1\right) I+\left(r_{1}-a-1\right) J .
$$

Let $S=r_{1}-1$, the line sum of $W$. Consider the principal submatrix $B$ of $W$ through the columns with ones in row one. This $S \times S$ matrix $B$ has column sums $S-2$ and trace zero. So $B$ has a row with sum at least $S-2$ and this row will meet the corresponding column of $B$ in at least $S-3$ positions. Thus $d_{1}+S-1 \geqq S-3$ or $d_{1} \geqq-2$. Since $d_{1}>1$ would force corresponding hits in $W$ to exceed $S$ we deduce

$$
-2 \leqq d_{1} \leqq 1
$$

Now with $d_{1}=-2$ we have that $B$ has line sums $S-2$ and row $i$ of $B$ hits column $i$ of $Z$ for $2 \leqq i \leqq S+1$ in a minimum of $S-3$ positions. Since now $d_{1}+S-1=S-3$ we conclude that column one of $Z$ has zeros in positions 2 through $S+1$ forcing row 1 to miss column 1 and $d_{1}+s-1=0$ so $S=3$. One then easily eliminates $a=0$ and $a=3$ and $a=2$ forces $W^{2}=J-I$ of order 10. But $J-I$ of order 10 has a negative determinant, so we are left with $a=1$, $n_{1}=7$ and the matrix IVe pops up unique to within equivalence.

We proceed with the cases on $d_{1}$ according to (3.20). If $d_{1}=-1$ (3.19) becomes

$$
W^{2}+(2-a) W=(a-2) I+(S-a) J
$$

with

$$
\left(n_{1}-S-1\right)(S-a)=S+2 .
$$

The eigenvalues of $W$ are then $S$ of multiplicity one $(S \neq a$ so $W$ is irreducible) and then the roots of $x^{2}+(2-a) x+(2-a)=0$. These roots are $\left.(a-2) \pm \sqrt{a^{2}-4}\right) / 2$. For $a \neq 2$ these are irrational or imaginary so that trace $W=0$ means $n_{1}$ is odd and

$$
S+\frac{n_{1}-1}{2}(a-2)=0 \text {. }
$$

Then $a=0,1$ with $S=n_{1}-1, n_{1}-1 / 2$ respectively. The former gives $Z_{1}=J$ earlier eliminated and the latter is incompatible with (3.22). In case $\mathrm{r}=2$ we have $W^{2}=(S-2) J$. The eigenvalues of $W$ are then $S$ and 0 denying trace $W=0$.

The case $d_{1}=+1$ is similarly eliminated as follows. The eigen- 
values of $W$ other than $S$ are

$$
\frac{a-2 \pm \sqrt{a^{2}+4}}{2}
$$

For $a \neq 0$ these are not rational. Hence $n_{1}$ is odd and $S+\left(n_{1}-1\right) / 2(a-2)=$ 0 , yielding a $=1, S=\left(n_{1}-1\right) / 2$. This is incompatible with $S^{2}+(2-a) S=$ $d_{1}+S-1+\left(n_{1}-1\right)(S-a)$ unless $S=2, n_{1}=5, W^{2}+W=(I+J)_{5}$ yielding the matrix (iva). In case $a=0$ we have $W^{2}+2 W=S J$, $S^{2}+2 S=n_{1} S$ so $S=n_{1}-2, r_{1}=n_{1}-1$ and $Z$ is regular.

The final case is $d_{1}=0$. Here the above techniques fail as the spectrum of $W$ is $\{a-1,-1, S\}$ with appropriate multiplicities. So consider the structure of $W$ :

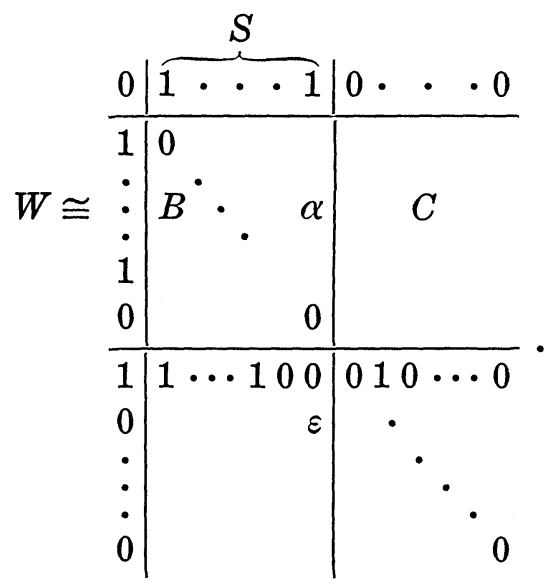

It is not difficult to see that $W$ has the structure of (3.23). The $(S+2)$ rd row being obtained by considering its inner product with column one. Now investigating this row and column $S+1$ recalling that $B$ has column sums $S-2$ we have

$$
1+\tau+\varepsilon=S-a .
$$

Where $\varepsilon=0,1, \tau=S-2, S-3$ depending on whether $\alpha$ is zero or one. In any case (3.24) shows $a=0,1,2$. From our remaks about the spectrum of $W$ it follows that if $a=0 n_{1}=S+1$ and $Z_{1}=J^{1}$. If $a=1$ we have $W^{2}+W=(S-1) T$ so $S^{2}+S=n_{1}(S-1)$ yielding

$$
S=\frac{\left(n_{1}-1\right) \pm \sqrt{\left(n_{1}-3\right)^{2}-8}}{2}
$$

From (3.25) and the fact that $S$ is a nonnegative integer with $S<n_{1}-1$ we conclude that $n_{1}=6, S=2,3$. These parameters give cases (ivd) and (ivf).

$1 \Lambda(W)=\{S,-1, a-1\}$, if $m$ is the multiplicity of $a-1=-1$ we have $S-$ $m-\left(n_{1}-m-1\right)=0$ or $S=n_{1}-1$. 
Finally for $a=2$ we have

$$
W^{2}=I+(S-2) J \text { with } S^{2}=1+n_{1}(S-2)
$$

or

$$
S=\frac{n_{1} \pm \sqrt{\left(n_{1}-4\right)^{2}-12}}{2}
$$

Then (3.26) forces $n_{1}=8, S=3,5$. In case $S=3$ one obtains the matrix (iii) and the case $S=5$ violates $a \leqq n_{1}-S-2$ easily seen to be necessary from (3.23) since the matrix $C$ has column $S-a$.

We remark in conclusion that the various matrices in Theorem 3.3 are easily seen to be non-equivalent by considerations of parameters and trace.

\section{REFERENCES}

1. W. G. Bridges and H. J. Ryser, Combinatorial designs and related systems, J. Algebra, 13 (1969), 423-446.

2. T. Evans, Products of points-some simple algebras and their identities, Amer, Math. Monthly, 74 (1967), 362-372.

3. A. J. Hoffman, On the polynomial of a graph, Amer. Math. Monthly, 70 (1963), $30-36$.

4. A. J. Hoffman and M. H. McAndrew, On the polynomial of a directed graph, Proc. Amer. Math. Soc.. 10 (1965), 303-309.

5. A. J. Hoffman and R. R. Singleton, On Moore Graphs of diameter 2 and 3, I.B.M.

J. Res. and Dev., 4 (Nov. 1960).

6. D. Knuth, Notes on central groupoids, (to appear).

7. H. J. Ryser, Combinatorial Mathematics (Carus Math. Monograph No. 14, Math. Assoc. Amer.) Wiley, New York, 1963.

8. - A generalization of the matrix equation $A^{2}=J$, J. Linear Algebra and Applications (to appear).

9. J. J. Seidel, Strongly regular graphs with $(-1,1,0)$ adjacency matrix having eigenvalue 3, Linear Alg. Applications, 1 (1968), 281-298.

10. J. J. Seidel, Strongly regular graphs of L-type and of triangular type, Koninkl. Ned. Akad. Wetenschap. Proc. Ser A 70 (1967), 188-196.

11. - Strongly regular graphs (Recent Progress in Combinatorics) Academic Press, New York 1969.

12. J. J. Seidel and J. M. Goethals, Strongly regular graphs derived from combinatorial designs, Can. J. Math. (to appear).

Received October 6, 1970 and in revised form March 16, 1971. This research was supported in part by ARO contract DA 131-124-ARO(D)-336.

City UNIVERSITY OF NEW YORK

AND

The UNIVERsity oF WYoming 



\section{PACIFIC JOURNAL OF MATHEMATICS}

\section{EDITORS}

\author{
H. SAMELSON \\ Stanford University \\ Stanford, California 94305 \\ C. R. HовBY \\ University of Washington \\ Seattle, Washington 98105
}

J. DUGUndis

Department of Mathematics

University of Southern California

Los Angeles, California 90007

RICHARD ARENS

University of California

Los Angeles, California 90024

\section{ASSOCIATE EDITORS}
E. F. BeCKenbaCH
B. H. NeUmanN
F. WOLF
K. YoshidA

\section{SUPPORTING INSTITUTIONS}

\author{
UNIVERSITY OF BRITISH COLUMBIA \\ CALIFORNIA INSTITUTE OF TECHNOLOGY \\ UNIVERSITY OF CALIFORNIA \\ MONTANA STATE UNIVERSITY \\ UNIVERSITY OF NEVADA \\ NEW MEXICO STATE UNIVERSITY \\ OREGON STATE UNIVERSITY \\ UNIVERSITY OF OREGON \\ OSAKA UNIVERSITY \\ UNIVERSITY OF SOUTHERN CALIFORNIA
}

\author{
STANFORD UNIVERSITY \\ UNIVERSITY OF TOKYO \\ UNIVERSITY OF UTAH \\ WASHINGTON STATE UNIVERSITY \\ UNIVERSITY OF WASHINGTON

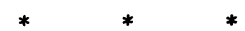 \\ AMERICAN MATHEMATICAL SOCIETY \\ CHEVRON RESEARCH CORPORATION \\ NAVAL WEAPONS CENTER
}

The Supporting Institutions listed above contribute to the cost of publication of this Journal, but they are not owners or publishers and have no responsibility for its content or policies.

Mathematical papers intended for publication in the Pacific Journal of Mathematics should be in typed form or offset-reproduced, (not dittoed), double spaced with large margins. Underline Greek letters in red, German in green, and script in blue. The first paragraph or two must be capable of being used separately as a synopsis of the entire paper. The editorial "we" must not be used in the synopsis, and items of the bibliography should not be cited there unless absolutely necessary, in which case they must be identified by author and Journal, rather than by item number. Manuscripts, in duplicate if possible, may be sent to any one of the four editors. Please classify according to the scheme of Math. Rev. Index to Vol. 39. All other communications to the editors should be addressed to the managing editor, Richard Arens, University of California, Los Angeles, California, 90024.

50 reprints are provided free for each article; additional copies may be obtained at cost in multiples of 50 .

The Pacific Journal of Mathematics is published monthly. Effective with Volume 16 the price per volume (3 numbers) is $\$ 8.00$; single issues, $\$ 3.00$. Special price for current issues to individual faculty members of supporting institutions and to individual members of the American Mathematical Society: $\$ 4.00$ per volume; single issues $\$ 1.50$. Back numbers are available.

Subscriptions, orders for back numbers, and changes of address should be sent to Pacific Journal of Mathematics, 103 Highland Boulevard, Berkeley, California, 94708.

PUBLISHED BY PACIFIC JOURNAL OF MATHEMATICS, A NON-PROFIT CORPORATION

Printed at Kokusai Bunken Insatsusha (International Academic Printing Co., Ltd.), 7-17, Fujimi 2-chome, Chiyoda-ku, Tokyo, Japan. 


\section{Pacific Journal of Mathematics}

Vol. 38, No. $2 \quad$ April, 1971

Richard Davis Anderson and Thomas Ashland Chapman, Extending

homeomorphisms to Hilbert cube manifolds .................. 281

Nguyen Huu Anh, Restriction of the principal series of $\operatorname{SL}(n, \mathbf{C})$ to some

reductive subgroups................................ 295

David W. Boyd, Indices for the Orlicz spaces . . . . . . . . . . . . 315

William Garfield Bridges, The polynomial of a non-regular digraph ...... 325

Billie Chandler Carlson, Robert K. Meany and Stuart Alan Nelson, Mixed

arithmetic and geometric means........................ 343

H. A. Çelik, Commutative associative rings and anti-flexible rings ...... 351

Hsin Chu, On the structure of almost periodic transformation groups ...... 359

David Allyn Drake, The translation groups of n-uniform translation

Hjelmslev planes ................................ 365

Michael Benton Freeman, The polynomial hull of a thin two-manifold . . . . 377

Anthony Alfred Gioia and Donald Goldsmith, Convolutions of arithmetic

functions over cohesive basic sequences .................... 391

Leslie C. Glaser, A proof of the most general polyhedral Schoenflies

conjecture possible ................................

Thomas Lee Hayden and Ted Joe Suffridge, Biholomorphic maps in Hilbert

space have a fixed point ................................ 419

Roger Alan Horn, Schlicht mappings and infinitely divisible kernels ...... 423

Norman Ray Howes, On completeness ...................... 431

Hideo Imai, Sario potentials on Riemannian spaces................ 441

A. A. Iskander, Subalgebra systems of powers of partial universal

algebras.

Barry E. Johnson, Norms of derivations of $\mathscr{L}(\mathrm{X})$.

David Clifford Kay and Eugene W. Womble, Axiomatic convexity theory and relationships between the Carathéodory, Helly, and Radon numbers

Constantine G. Lascarides, A study of certain sequence spaces of Maddox

and a generalization of a theorem of Iyer .............

C. N. Linden, On Blaschke products of restricted growth .

John S. Lowndes, Some triple integral equations ................. 515

Declan McCartan, Bicontinuous preordered topological spaces ......... 523

S. Moedomo and J. Jerry Uhl, Jr., Radon-Nikodým theorems for the Bochner and Pettis integrals ...

Calvin Cooper Moore and Joseph Albert Wolf, Totally real representations

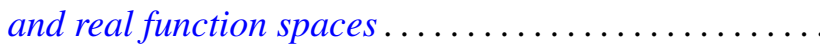

Reese Trego Prosser, A form of the moment problem for Lie groups. ... 